\title{
Adaptabilidade e estabilidade de genótipos eretos de amendoim cultivados nas regiões Nordeste e Centro-Oeste
}

\author{
Comparison of adaptability and stability estimates in upright peanut genotypes cultivated in \\ Northeast and Midwest regions
}

\section{Felipe Matheus Teles de Vasconcelos ${ }^{I}$ Ramom Araújo de Vasconcelos ${ }^{\text {II }}$ Lucas Nunes da Luz ${ }^{\text {III }}$ Norival Thiago Cabral ${ }^{\mathrm{IV}}$ João Oscar Lustosa de Oliveira Júnior ${ }^{\mathrm{V}}$ Antônio Dias Santiago ${ }^{\mathrm{VI}}$ Edvaldo Sgrillo $^{\mathrm{V}}$ Francisco José Correia Farias $^{\mathrm{II}}$ Péricles de Albuquerque Melo Filho ${ }^{\mathrm{I}}$ Roseane Cavalcanti dos Santos $^{\mathrm{II}^{*}}$}

\section{RESUMO}

\begin{abstract}
$O$ presente trabalho teve por objetivo estimar a estabilidade e a adaptabilidade de oito genótipos de amendoim ereto e comparar os modelos de análise de EBERHART \& RUSSELL (1966), WRICKE (1965) e LIN \& BINNS (1988), baseando-se em dados de produtividade de vagens e sementes. Os genótipos de amendoim foram avaliados em 14 ambientes situados nas Regioes Nordeste e Centro-Oeste, durante os anos de 2006 a 2011, sob o delineamento de blocos ao acaso com cinco repetições. Verificou-se que, entre os três métodos estudados, os de EBERHART \& RUSSELL(1966) e LIN \& BINNS (1988) geraram informações concordantes para estimativas de adaptação e estabilidade de produção de vagens e sementes, baseando-se nas condições deste estudo. Os genótipos L7 Bege e BRS 151 L7 foram os mais produtivos, com adaptabilidade especifica a ambientes favoráveis, enquanto que CNPA 280 revelou adaptabilidade ampla e alta estabilidade fenotípica.
\end{abstract}

Palavras-chave: Arachis hypogaea, interação genótipo $x$ ambiente, produtividade, semiárido, cerrados.

\section{ABSTRACT}

This study aimed to estimate the stability and adaptability parameters in upright-peanut genotypes, based on pod and seed yield, through EBERHART \& RUSSELL (1966), WRICKE (1965) and LIN \& BINNS (1988) methodologies. The genotypes were evaluated in 14 environments located in Northeast and Midwest regions, during 2006 to 2011, in a randomized blocks design, with five replications. EBERHART \& RUSSELL (1966) and LIN \& BINNS (1988) methodologies revealed similar results, identifying genotypes with high adaptability and stability to pod and seed yield. The BRS 151 L7 and L7 Bege were the most productive, revealing specific adaptability to favorable environment, while CNPA 280 AM revealed broad adaptability and high phenotypical stability.

Key words: Arachis hypogaea, genotype $x$ environment interaction, yield, arid zone, savanna.

\section{INTRODUÇÃO}

O amendoim (Arachis hypogaea L.) é uma oleaginosa de largo aproveitamento alimentar, atendendo os vários segmentos de consumo in natura ou industrializado. Mais recentemente, com as novas tendências no segmento de fontes renováveis, o amendoim tem sido também demandado para atender os mercados de óleo comestível e combustível. A produção nacional é de 300 mil toneladas anuais (IBGE, 2012), sendo, em média, 80\% destas, produzidas na região Sudeste do país.

Nos últimos anos, o cultivo do amendoim tem crescido na região Nordeste, devido à adoção de cultivares precoces e tolerantes à baixa disponibilidade hídrica. Elas são adaptadas ao semiárido, pertencentes à Embrapa Algodão, para diferentes estados e regiões do semiárido. Contudo, a diversificação climática do semiárido, que engloba desde climas úmidos a semiáridos, contribui para

IUniversidade Federal Rural de Pernambuco (UFRPE), Recife, PE, Brasil.

IIEmbrapa Algodão, CP 174, 58428-095, Campina Grande, PB, Brasil. E-mail: roseane.santos@embrapa.br. "Autor para correspondência.

IIIniversidade Federal do Cariri (UFCA), Crato, CE, Brasil.

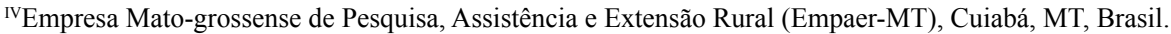

vEmbrapa Meio Norte, Teresina, PI, Brasil.

${ }^{\mathrm{VI} E m b r a p a}$ Tabuleiros Costeiros, Aracaju, SE, Brasil. 
altas estimativas de interação genótipo $\mathrm{x}$ ambiente (GA) nas populações em fase de melhoramento, dificultando tanto a seleção quanto a identificação de genótipos com estabilidade de produção.

Os efeitos da interação GA na seleção de genótipos dificilmente são nulos, porém, podem ser minimizados em função do maior número de ensaios, repetidos em vários locais e anos, o que permitirá maior confiabilidade nos processos de seleção e posterior indicação do genótipo mais promissor, em termos de produtividade e adaptabilidade (SILVA \& DUARTE, 2006; SANTOS et al., 2012). Várias metodologias estão disponíveis na literatura para estimar a estabilidade e adaptabilidade dos genótipos a vários locais. Entre eles, destacam-se os fundamentados na análise de variância WRICKE (1965), na regressão linear simples EBERHART \& RUSSELL (1966); na regressão linear bissegmentada CRUZ et al. (1989); e métodos não paramétricos LIN \& BINNS (1988), entre outros.

A metodologia proposta por EBERHART \& RUSSELL (1966) é uma das mais estudadas na avaliação da adaptabilidade e estabilidade fenotípica dos genótipos e é baseada na regressão linear simples dos genótipos em função dos índices ambientais. Estudos de estabilidade e adaptabilidade do amendoim utilizando a análise de regressão já foram utilizados em vários trabalhos (MEKONTCHOU et al., 2006; OLIVEIRA et al., 2006; OLIVEIRA \& GODOY, 2006; GOMES et al., 2007; SANTOS et al.,2012). Do mesmo modo, a estimativa de Ecovalência $\left(\omega_{\mathrm{i}}\right)$ WRICKE (1965), estimada através da decomposição da soma de quadrados da interação GA, e o índice de estabilidade $\left(\mathrm{P}_{\mathrm{i}}\right)$ LIN \& BINNS (1988) são boas alternativas para aferir a reposta ambiental dos genótipos avaliados (RAMALHO et al., 1993; FARIAS et al., 1997; SILVA FILHO et al., 2008).

Neste trabalho, foram estimados os parâmetros de adaptabilidade e estabilidade de genótipos de amendoim ereto, adotando-se os modelos de EBERHART \& RUSSELL(1966), WRICKE (1965) e LIN \& BINNS (1988), baseandose em dados de produtividade de vagens e sementes, visando a selecionar os mais adaptados às condições das regiões Nordeste e Centro Oeste.

\section{MATERIAL E MÉTODOS}

Oito genótipos de amendoim de porte ereto (três cultivares e cinco linhagens avançadas) foram avaliados em 14 ambientes situados nas Regiões Nordeste e Centro-Oeste, durante os anos de 2006 a 2011, no período da estação chuvosa de cada local (Tabela 1).

As parcelas foram constituídas de três fileiras de $6 \mathrm{~m}$, espaçadas em $0.7 \mathrm{~m}$. O espaçamento entre plantas foi de $0.2 \mathrm{~m}$, deixando-se apenas duas plantas/cova. A fileira central foi considerada área útil para coleta dos dados. O delineamento experimental adotado foi blocos ao acaso com cinco repetições. O manejo dos genótipos seguiu de acordo com as recomendações de SANTOS et al. (2006). Não foi procedido nenhum controle fitossanitário contra doenças. A colheita foi iniciada aos 85 dias para os genótipos mais precoces (BR 1, BRS Havana, BRS 151 L 7 e L7 bege) e aos 95 dias para CNPA 270, CNPA 271, CNPA 280 e CNPA

Tabela 1 - Coordenadas geográficas e características edafoclimáticas dos municípios avaliados.

\begin{tabular}{|c|c|c|c|c|c|c|}
\hline Ambientes & Latitude & Longitude & Altitude (m) & Precipitação ${ }^{1}$ anual (mm) & Solo $^{2}$ & Clima \\
\hline Parnamirim - PE & $8^{\circ} 5^{\prime} 27^{\prime \prime} \mathrm{S}$ & $39^{\circ} 34^{\prime} 40^{\prime \prime} \mathrm{W}$ & 392 & 432 & Oxisolo & Semiárido \\
\hline Araripina - PE & $7^{\circ} 34^{\prime} 33^{\prime \prime} \mathrm{S}$ & $40^{\circ} 29^{\prime} 52^{\prime \prime} \mathrm{W}$ & 622 & 615 & Vertissolo & Semiárido \\
\hline Lagoa de Dentro - PB & $6^{\circ} 40^{\prime} 22^{\prime \prime} \mathrm{S}$ & $35^{\circ} 22^{\prime} 44^{\prime \prime} \mathrm{W}$ & 154 & 432 & Entisolo & Tropical \\
\hline Goiana - PE & $7^{\circ} 33^{\prime} 39^{\prime \prime} \mathrm{S}$ & $35^{\circ} 00^{\prime} 10^{\prime \prime} \mathrm{W}$ & 80 & 568 & Entisolo & Tropical \\
\hline Monteiro - PB & $7^{\circ} 53^{\prime} 20^{\prime \prime} \mathrm{S}$ & $37^{\circ} 07^{\prime} 12^{\prime \prime} \mathrm{W}$ & 599 & 620 & Oxisolo & Semiárido \\
\hline Itaporanga-PB & $7^{\circ} 18^{\prime} 14^{\prime \prime} \mathrm{S}$ & $38^{\circ} 09^{\prime} 00^{\prime \prime} \mathrm{W}$ & 291 & 1634 & Entisolo & Desértico \\
\hline Coruripe - AL & $10^{\circ} 7^{\prime} 33^{\prime \prime} \mathrm{S}$ & $36^{\circ} 10^{\prime} 33^{\prime \prime} \mathrm{W}$ & 36 & 1630 & Vertisolo & Tropical \\
\hline São João do Piauí - PI & $8^{\circ} 21^{\prime} 28^{\prime \prime} \mathrm{S}$ & $42^{\circ} 14^{\prime} 49^{\prime \prime} \mathrm{W}$ & 235 & 763 & Oxisolo & Semiárido \\
\hline Colinas - MA & $6^{\circ} 01^{\prime} 33^{\prime \prime} \mathrm{S}$ & $44^{\circ} 14^{\prime} 57^{\prime \prime} \mathrm{W}$ & 180 & 1120 & Entisolo & Tropical \\
\hline Barbalha - CE & $7^{\circ} 18^{\prime} 18^{\prime \prime} \mathrm{S}$ & $39^{\circ} 18^{\prime} 07^{\prime \prime} \mathrm{W}$ & 414 & 1160 & Vertisolo & Semiárido \\
\hline S. J. dos 4 Marcos - MT & $15^{\circ} 39^{\prime} 11^{\prime \prime} \mathrm{S}$ & $58^{\circ} 18^{\prime} 56^{\prime \prime} \mathrm{W}$ & 280 & 1049 & AVAE & Cerrados \\
\hline Tangará da Serra - MT & $14^{\circ} 37^{\prime} 8^{\prime \prime} \mathrm{S}$ & $57^{\circ} 29^{\prime} 09^{\prime \prime} \mathrm{W}$ & 423 & 1448 & LVDT & Cerrados \\
\hline Sinop - MT & $11^{\circ} 58^{\prime} 53^{\prime \prime} \mathrm{S}$ & $55^{\circ} 33^{\prime} 51^{\prime \prime} \mathrm{W}$ & 384 & 2500 & LVAD & Cerrados \\
\hline
\end{tabular}

${ }^{1}$ Média histórica, ${ }^{2}$ AVAE- Argissolo Vermelho Amarelo Eutrófico, LVDT- Latossolo Vermelho Distrófico Típico; LVAD- Latossolo Vermelho Amarelo Distrófico. 
283. As variáveis analisadas foram produtividade em vagens e em sementes.

Após obtenção dos dados, foram procedidas as análises estatísticas, utilizando-se o programa Genes, versão 2009.7.0 (CRUZ, 2006). Para estimativa de estabilidade e adaptabilidade, foram adotados os métodos propostos por WRICKE (1965), EBERHART \& RUSSELL (1966) e LIN \& BINNS (1988).

O método proposto por WRICKE (1965) estima a ecovalência $\left(\omega_{\mathrm{i}}\right)$, que é obtida por:

$\omega=r \sum_{\mathrm{J}}\left[{\overline{Y_{1 j}}}_{1 \mathrm{Y}}-\overline{\mathrm{Y}}_{\mathrm{j}}-\overline{\mathrm{Y}} \ldots\right]^{2}$ em que ${ }_{\mathrm{ij}}$ é a média do genótipo i no ambiente $\mathrm{j}$; $\mathrm{i}$ é a média do genótipo $i,{ }_{j}$ a média do ambiente $\mathrm{j}$ e a média geral de todos os genótipos em todos os ambientes. Os genótipos mais estáveis são aqueles com menores valores de $\omega_{\mathrm{i}}$ (RAMALHO et al., 1993).O método de EBERHART \& RUSSELL (1966) é baseado na regressão linear, obtida por $Y_{i j}=\mu+\beta_{i} I_{j}+\delta_{i j}+\varepsilon_{i j}$, em que ${ }_{i j}$ é o comportamento do genótipo i no ambiente $\mathrm{j} ; \mu$ é a média geral; $\beta_{\mathrm{i}}$ é o coeficiente de regressão linear; I é o índice ambiental; $\delta_{\mathrm{ij}}$ é o desvio da regressão do genótipo i no ambiente $\mathrm{j}$; e $\varepsilon_{\mathrm{ij}}$ o erro associado a média. Conforme esse modelo, a adaptabilidade de um genótipo é estimada pelo seu coeficiente de regressão $\left(\beta_{\mathrm{i}}\right)$ em relação ao índice ambiental $\left(\mathrm{I}_{\mathrm{j}}\right)$ de cada ambiente, e a estabilidade pela variância dos desvios de regressão $\sigma_{\text {di. }}^{2}$ Consideram-se, portanto, como estáveis, os genótipos que apresentarem $\beta_{\mathrm{i}}$ igual a 1 e $\sigma_{\text {di }}^{2}$ que não difira significativamente de zero. A análise não paramétrica de adaptabilidade e estabilidade conforme o método proposto LINN \& BINNS (1988) é baseada no índice $\mathrm{Pi}=\Sigma\left(\mathrm{Y}_{\mathrm{ij}}-\mathrm{M}_{\mathrm{j}}\right)^{2} / 2 \mathrm{n}$, em que $\mathrm{P}_{\mathrm{i}}$ é a estimativa do índice de estabilidade do genótipo i; $\mathrm{Y}_{\mathrm{ij}}$ é o comportamento do genótipo i no ambiente $\mathrm{j} ; \mathrm{M}_{\mathrm{j}}$ é a resposta máxima observada entre todos os genótipos no ambiente $\mathrm{j}$; e n é o número de ambientes. $\mathrm{O}$ índice $\mathrm{P}_{\mathrm{i}}$ indica a estabilidade de um genótipo e, quanto menor for o seu valor, mais estável é o material. Os três métodos foram comparados utilizando-se a correlação de Spearman, baseandose na média geral dos genótipos, no coeficiente de regressão $\left(\beta_{\mathrm{i}}\right)$, na variância dos desvios da regressão $\left(\sigma_{\text {di }}^{2}\right)$, na ecovalência $\left(\omega_{i}\right)$ e nos valores de $P_{i}$.

\section{RESULTADOS E DISCUSSÃO}

Verificou-se diferença estatística significativa entre genótipos, entre ambientes e efeitos de interação genótipos x ambientes (Tabela 2), indicando resposta diferenciada em função dos ambientes estudados. Os coeficientes de variação experimental obtidos foram de $15.90 \%$ e $17.84 \%$ para produtividades de vagens e sementes, respectivamente.

As estimativas dos parâmetros de adaptabilidade e estabilidade fenotípica nos três métodos avaliados encontram-se nas tabelas $3 \mathrm{e}$ 4 para produtividade de vagens e de sementes. Para produtividade de vagens, observou-se que os genótipos L7 Bege, BRS 151 L7 e BR 1 apresentaram os maiores rendimentos, superando a média geral em $13 \%, 7 \%$ e $4 \%$, respectivamente (Tabela 3). Considerando-se a estabilidade dos genótipos, verificou-se, pelo método proposto por WRICKE (1965), que BRS $151 \mathrm{~L} 7\left(\omega_{\mathrm{i}}=27.00 \times 10^{5}\right)$ e CNPA $280\left(\omega_{i}=35.64 \times 10^{5}\right)$ foram mais estáveis, com menores estimativas de Ecovalência. Pela metodologia de LIN \& BINNS (1988), as menores estimativas de $\mathrm{P}_{\mathrm{i}}$ ocorreram para os genótipos CNPA L7 Bege $\left(\mathrm{P}_{\mathrm{i}}=2.53\right)$ e BRS 151 L7 $\left(\mathrm{P}_{\mathrm{i}}=11.36\right)$. Estes genótipos apresentaram elevadas médias de produtividade e pequena contribuição para a interação GA, com valores inferiores a $45 \%$.

Tabela 2 - Resumo da análise de variância conjunta para as variáveis produtividade de vagens (PV) e sementes (PS) em oito genótipos de amendoim, cultivados em 14 ambientes.

\begin{tabular}{llll}
\hline & & & \\
Fonte de variação & GL & PV & PS \\
\hline Bloco/Ambiente & 56 & $317470.91^{* *}$ & $192414.69 * *$ \\
Genótipo (G) & 7 & $3650815.18^{* *}$ & $2050249.78^{* *}$ \\
Ambiente (A) & 13 & $18571809.91^{* *}$ & $9468401.33^{* *}$ \\
Interação GA & $91(56)$ & $786580.12^{* *}$ & $456013.44 *$ \\
Resíduo & $(235)$ & 185369.41 & 107110.76 \\
Média & & 2707.36 & 1834.74 \\
CV(\%) & & 15.90 & 17.84 \\
\hline
\end{tabular}

GL- grau de liberdade. Dados entre parênteses representam o grau de liberdade, corrigido pelo método de COCHRAN (1954), em razão da heterocedasticidade. $*$ e ** Significativo a 5 e $1 \%$ de probabilidade, respectivamente, pelo teste $\mathrm{F}$. 


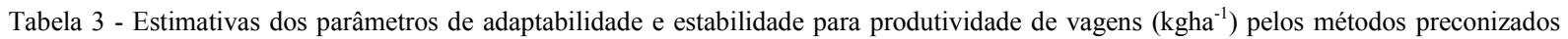
por WRICKE (1965) e EBERHART \& RUSSELL (1966) e pelo índice de LIN \& BINNS (1988), em genótipos de amendoim avaliados em 14 ambientes da região Nordeste e Centro-Oeste, no período de 2006 a 2011.

\begin{tabular}{|c|c|c|c|c|c|c|c|c|c|}
\hline \multirow{2}{*}{ Genótipos } & \multirow{2}{*}{ Média } & \multirow{2}{*}{ Wricke $\left(\omega_{\mathrm{i}}\right)\left(10^{5}\right)$} & \multicolumn{4}{|c|}{ 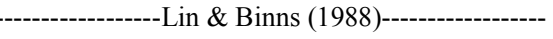 } & \multicolumn{3}{|c|}{-------Eberhart \& Russell (1966) ----- } \\
\hline & & & $P_{i}$ geral $\left(\times 10^{4}\right)$ & DG & DI & CPI $(\%)$ & $\beta_{\mathrm{i}}$ & $\sigma_{\mathrm{di}}^{2}\left(\mathrm{x} 10^{4}\right)$ & $\mathrm{R}^{2}(\%)$ \\
\hline CNPA 270 & $2452(7)$ & $46.7(3)$ & $38.92(7)$ & 28.40 & 10.52 & 72.97 & 0.96 & $5.49 * *$ & 85.83 \\
\hline CNPA 271 & $2356(8)$ & $49.1(4)$ & $49.80(8)$ & 36.08 & 13.72 & 72.45 & $0.87 *$ & $5.11 * *$ & 83.83 \\
\hline CNPA 280 & $2685(5)$ & $35.64(2)$ & $21.75(5)$ & 13.55 & 8.2 & 62.33 & 1.02 & $3.69 * *$ & 89.86 \\
\hline CNPA 283 & $2717(4)$ & $52.8(5)$ & 20.55 (4) & 11.94 & 8.61 & 58.09 & 0.99 & $6.57 * *$ & 84.78 \\
\hline L7 Bege & 3065 (1) & $102.3(8)$ & $2.53(1)$ & 0.99 & 1.54 & 39.02 & $1.18^{* *}$ & $13.19 * *$ & 81.98 \\
\hline BRS Havana & $2670(6)$ & $60.2(6)$ & $25.05(6)$ & 14.37 & 10.67 & 57.38 & 0.91 & $7.40 * *$ & 81.23 \\
\hline BR 1 & $2826(3)$ & $66.7(7)$ & $16.77(3)$ & 7.21 & 9.56 & 43.02 & 1.04 & $8.82 * *$ & 83.08 \\
\hline BRS 151 L7 & $2888(2)$ & $27.0(1)$ & $11.36(2)$ & 5.06 & 6.30 & 44.51 & 1.03 & $2.23 *$ & 92.30 \\
\hline
\end{tabular}

DG- desvio genético, DI- desvio da interação, CPI- contribuição para interação, * e ** Significativo a 5 e $1 \%$ de probabilidade, respectivamente, pelo teste de $\mathrm{t}\left(\beta_{\mathrm{i}}\right)$ e $\mathrm{F}\left(\sigma_{\mathrm{di}}^{2}\right)$.

Pelo método de EBERHART \& RUSSELL (1966), todos os genótipos, com exceção do CNPA 271 e do L7 Bege, possuem adaptabilidade ampla; o genótipo L7 Bege apresentou estimativas de $\beta_{i}$ superior à unidade, indicando adaptabilidade específica para ambientes favoráveis, enquanto que CNPA 271 revelou adaptabilidade específica para ambientes desfavoráveis. Quanto à estabilidade, nenhum genótipo foi estável para a variável produção de vagens, considerando-se a variância dos desvios da regressão. Contudo, analisando-se o coeficiente de determinação $\left(\mathrm{R}^{2}\right)$, que estima a previsibilidade do genótipo nos vários ambientes, observa-se que BRS 151 L 7 foi a mais estável, com $\mathrm{R}^{2}$ superior a $90 \%$. De acordo com PINTHUS (1973), esse parâmetro pode substituir ou auxiliar na interpretação da estabilidade nos casos de genótipos com boa média e variância dos desvios de regressão significativos, validando assim a cultivar como estável para as condições das regiões avaliadas.

Para a produtividade de sementes (Tabela 4), observou-se a mesma tendência de estabilidade pelos métodos propostos por WRICKE (1965) e de LIN \& BINNS (1988),

Tabela 4 - Estimativas dos parâmetros de adaptabilidade e estabilidade para produtividade de sementes $\left(\mathrm{kgha}^{-1}\right)$, de acordo com os métodos de WRICKE (1965), EBERHART \& RUSSELL (1966) e pelo índice de LIN \& BINNS (1988) em genótipos de amendoim, avaliados em 14 ambientes da região Nordeste e Centro-Oeste, no período de 2006 a 2011.

\begin{tabular}{|c|c|c|c|c|c|c|c|c|c|}
\hline \multirow{2}{*}{ Genótipos } & \multirow{2}{*}{ Média } & \multirow{2}{*}{$\begin{array}{l}\text { Wricke } \quad\left(\omega_{\mathrm{i}}\right) \\
\left(\mathrm{x} 10^{5}\right)\end{array}$} & \multicolumn{4}{|c|}{----------------Lin \& Binns (1988)---------------- } & \multicolumn{3}{|c|}{-------Eberhart \& Russell (1966)---- } \\
\hline & & & $\mathrm{P}_{\mathrm{i}}$ geral $\left(\mathrm{x} 10^{4}\right)$ & DG & DI & CPI $(\%)$ & $\beta_{\mathrm{i}}$ & $\sigma_{\mathrm{di}}^{2}\left(\mathrm{x} 10^{4}\right)$ & $\mathrm{R}^{2}(\%)$ \\
\hline CNPA 270 & $1651(7)$ & $25.5(3)$ & $23.8(7)$ & 17.20 & 6.60 & 72.25 & 0.91 & $2.81 * *$ & 83.97 \\
\hline CNPA 271 & $1567(8)$ & $22.2(2)$ & $30.1(8)$ & 22.51 & 7.62 & 74.71 & $0.80 * *$ & $1.46^{*}$ & 85.95 \\
\hline CNPA 280 & $1831(4)$ & $28.4(6)$ & $14.9(5)$ & 8.28 & 6.62 & 55.60 & 0.99 & $3.49 * *$ & 84.25 \\
\hline CNPA 283 & $1803(6)$ & $271(5)$ & $15.4(6)$ & 9.47 & 5.97 & 61.29 & 0.94 & $3.18 * *$ & 83.65 \\
\hline L7 Bege & 2087 (1) & $56.5(8)$ & $3.3(1)$ & 1.13 & 2.16 & 34.41 & $1.26 * *$ & $6.44 * *$ & 84.17 \\
\hline BRS Havana & $1809(5)$ & $25.9(4)$ & $14.8(4)$ & 9.17 & 5.61 & 62.07 & 0.96 & $3.04 * *$ & 84.68 \\
\hline BR 1 & $1956(3)$ & $49.9(7)$ & $8.9(3)$ & 3.98 & 4.88 & 44.94 & 1.06 & $6.97 * *$ & 78.06 \\
\hline BRS 151 L7 & $1974(2)$ & $10.7(1)$ & $7.8(2)$ & 3.47 & 4.33 & 44.48 & 1.07 & 0.42 & 94.65 \\
\hline
\end{tabular}

DG- desvio genético. DI- desvio da interação, CPI- contribuição para interação, * e **significativo a 5 e $1 \%$ de probabilidade, respectivamente, pelo teste de $\mathrm{t}\left(\beta_{\mathrm{i}}\right)$ e $\mathrm{F}\left(\sigma_{\mathrm{di}}^{2}\right)$. 
como visto para a BRS 151 L7. O diferencial foi que, pelo método de EBERHART \& RUSSELL (1966), esta cultivar foi considerada mais estável, considerando-se tanto a não significância da variância dos desvios da regressão, quanto o alto valor de $\mathrm{R}^{2}(94.65 \%)$. Nota-se ainda que o genótipo L7 Bege foi altamente produtivo (14\% superior à média do experimento) e com adaptabilidade específica a ambientes favoráveis, sendo, entre os demais do grupo, o menos influenciado pelo efeito da interação GA, considerando-se os parâmetros de estabilidade propostos por LIN \& BINNS (1988).

Os resultados verificados neste trabalho, referentes à estabilidade da BRS $151 \mathrm{~L} 7$, tem sido demonstrada por outros autores. GOMES et al. (2007) estimaram a adaptabilidade e estabilidade dos genótipos deste estudo em oito ambientes do estado de Pernambuco, adotando o método de EBERHART \& RUSSELL (1966), e verificaram que BRS 151 L 7 comportou-se como a mais estável, sendo a recomendada para os ambientes estudados. Tal resultado condiz com a natureza genética da cultivar, que foi obtida por meio de cruzamentos entre genitores Brasileiro e Africano, o que lhe confere larga adaptação ao ambiente semiárido (SANTOS et al., 2012).

Considerando-se as três diferentes metodologias adotadas neste estudo, verificouse que os métodos de EBERHART \& RUSSELL (1966) e LIN \& BINNS (1988) foram concordantes, para ambas variáveis, conforme se observa por meio das estimativas da correlação de Spearman (Tabela 5). Essa tendência também tem sido observada em outros trabalhos, como os reportados por FARIAS et al. (1997) e SILVA FILHO et al. (2008) em algodão, SILVA \& DUARTE (2006) em soja, PEREIRA et al. (2009) em feijão, CONDÉ

Tabela 5 - Estimativas da correlação de Spearman entre os métodos utilizados para adaptabilidade e estabilidade na produção de vagens (PV) e de sementes (PS) de amendoim.

\begin{tabular}{lcccc}
\hline \multirow{2}{*}{ Métodos } & \multicolumn{1}{c}{----------PV-------- } & -----------PS----------- \\
& ER & W & ER & W \\
\hline LB & $0.902 * *$ & 0.285 & $0.912^{* *}$ & $0.571^{*}$ \\
ER & - & 0.285 & - & 0.476 \\
\hline
\end{tabular}

LB: LIN \& BINNS (1988), ER: EBERHART \& RUSSELL (1966), W: WRICKE (1965), PV: Produtividade em vagens, PS: Produtividade em sementes. et al.(2010) em trigo, entre outros. O método de WRICKE (1965) apresentou correlação mediana com o de LIN \& BINNS (1988), apenas para a variável produtividade de sementes.

A utilização de mais de um método para estimação dos parâmetros genéticos pelo melhorista é uma estratégia que permite maior confiabilidade na interpretação dos dados para posterior recomendação de linhas superiores ou cultivares para uma região. De acordo com SILVA \& DUARTE (2006), os métodos baseados exclusivamente em coeficientes de regressão devem ser utilizados em associação com outro, fundamentado na variância da interação GA. SCAPIM et al. (2010) estimaram a produção de grãos e capacidade de expansão de milho pipoca em 21 ambientes, utilizando os métodos de EBERHART \& RUSSELL (1966), WRICKE (1965), HUEHN (1990), LIN \& BINNS (1988) e a classificação de soma de KANG (1988). Os autores verificaram que os métodos de EBERHART \& RUSSELL (1966), WRICKE(1965) e HUEHN (1990) se correlacionaram positivamente para a variável capacidade de expansão, indicando que qualquer um dos métodos é indicado para seleção dessa característica. Contudo, o de EBERHART \& RUSSELL (1966) foi negativamente correlacionado com o de LIN \& BINNS (1988) para produção de grãos, indicando que os genótipos mais adaptáveis tendem a ter menores estimativas de $\mathrm{P}_{\mathrm{i}}$.

\section{CONCLUSÃO}

Os métodos de EBERHART \& RUSSELL (1966) e LIN \& BINNS (1988) foram concordantes para as estimativas de adaptabilidade e estabilidade de produção de vagens e sementes de amendoim nas condições deste estudo. Os genótipos L7 Bege e BRS 151 L7 são os mais produtivos, e os mais estáveis nos ambientes estudados, podendo ser recomendados para a maioria dos ambientes avaliados. O genótipo CNPA 280 tem adaptabilidade ampla e alta estabilidade fenotípica nos ambientes estudados, podendo ser recomendado para a maioria dos ambientes avaliados.

\section{REFERÊNCIAS}

COCHRAN, W.G. Some methods for strengthening the common $\chi 2$ tests. Biometrics, v.10, p.417-451, 1954. Disponível em: $<\mathrm{http} / / /$ www.jstor.org/discover/10.2307/3001616?uid=2129\&uid=2\&uid=7 0\&uid=4\&sid=21103668391793 > . Acesso em: 28 fev. 2014.

CONDÉ, A.B.T. et al. Adaptabilidade e estabilidade de trigo sobre cultivo de sequeiro em Minas Gerais. Pesquisa Agropecuária Tropical, v.40, n.1, p.45-52, 2010. Disponível em: $\quad<$ http://www.redalyc.org/articulo.oa?id=253020192006 $>$. Acesso em: 21 fev. 2014. 
CRUZ, C.D. Programa genes biometria 1. Viçosa: UFV, 2006. 382p.

CRUZ, C.D. et al. An alternative approach to the stability analysis proposed by Silva and Barreto. Revista Brasileira de Genética, v.12, n.2, p.567-580, 1989. Disponível em: <http://www.gmb.org. br/Revistas/V12/v12a53.pdf >. Acesso em: 28 fev. 2014.

EBERHART, S.A.; RUSSELL, W.A. Stability parameters for comparing varieties. Crop Science, v.6, p.36-40, 1966. Disponível em: <https://dl.sciencesocieties.org/publications/cs/abstracts/6/1/ CS0060010036>. Acesso em: 21 fev. 2014. doi:10.2135/cropsci1 966.0011183X000600010011x.

EMBRAPA (EMPRESA BRASILEIRA DE PESQUISA AGROPECUÁRIA). Diagnóstico do Semi-árido. 2003. Disponível em: <http://www.cpatsa.embrapa.br>. Acesso em: 09 set. 2012.

FARIAS, F.J.C. et al. Parâmetros de estabilidade propostos por Linn e Binns (1988) comparados com o método da regressão. Pesquisa Agropecuária Brasileira, v.32, n.4, p.407-414, 1997. Disponível em: <http://seer.sct.embrapa.br/index.php/pab/article/ view/4657>. Acesso em: 21 fev. 2014.

GOMES, L.R. etal.Adaptabilidade e estabilidade fenotípica de genótipos de amendoim de porte ereto. Pesquisa Agropecuária Brasileira, v.42, n.7, p.985-989, 2007. Disponível em: <http://www.scielo.br/scielo.php ?pid=S0100204X2012000800012\&script=sci_arttext $>$. Acesso em: 21 fev. 2014. doi: 10.1590/S0100-204X2012000800012.

HUEHN, M. Non parametric measures of phenotypic stability Part I: theory. Euphytica, v.47, n.3, p.195-201, 1990. Disponível em: $<$ http://link.springer.com/article/10.1007/BF00024241\#page-1>. Acesso em: 21 fev. 2014. doi: 10.1007/BF00024241.

IBGE (INSTITUTO BRASILEIRO DE GEOGRAFIA E ESTATÍSTICA). Safra de Amendoim. 2012. Disponível em: <http:/ wwwibgegovbr/home/presidência/noticias/noticia_visualizaphp?id_ noticia=1819\&id_paginal>. Acesso em: 22 set. $20 \overline{12}$.

KANG, M.S. A rank-sum method for selecting high yielding stable corn genotypes. Cereal Res Commun, v.16, p.113-115, 1988. Disponível em: <http://www.akademiai.com/content/120427/?sor torder=asc $>$. Acesso em: 21 fev. 2014.

LIN, C.S.; BINNS, M.R. A superiority measure of cultivar performance for cultivar $\mathrm{x}$ location data. Canadian Journal of Plant Science, v.68, p.193-198, 1988. Disponível em: <http://pubs. aic.ca/doi/abs/10.4141/cjps88-018>. Acesso em: 21 fev. 2014.

MEKONTCHOU, T. et al. Stability analysis for yield and yield components of selected peanut breeding lines (Arachis hypogaea L.) in the North Province of Cameroon. Tropicultura, v.24, n.2, p.90-94, 2006. Disponível em: <http://www.tropicultura.org/text/ v24n2/90.pdf>. Acesso em: 21 fev. 2014.

OLIVEIRA, E.J; GODOY, I.J. Pod yield stability analysis of runner peanut lines using AMMI. Crop Breeding and Applied Biotechnology, v.6, n.4, p.311-317, 2006. Disponível em: $<$ http://www.sbmp.org.br/cbab/siscbab/modules/news/article. php?storyid=386>. Acesso em: 21 fev. 2014. doi: 10.12702/19847033.v06n04a09.
OLIVEIRA, E.J.et al. Adaptabilidade e estabilidade de genótipos de amendoim de porte rasteiro. Pesquisa Agropecuária Brasileira, v.41, n.8, p.1253-1260, 2006. Disponível em: <http://www.scielo. br/scielo.php?pid $=$ S0100-204X2006000800007\&script $=$ sci arttext>. Acesso em: 21 fev. 2014. doi: 10.1590/S0100204X2006000800007.

PEREIRA, H.S. et al. Adaptabilidade e estabilidade de genótipos de feijoeiro-comum com grãos tipo carioca na Região Central do Brasil. Pesquisa Agropecuária Brasileira, v.44, n.1, p.29-37, 2009. Disponível em: <http://www.scielo.br/scielo. php?script $=$ sci_arttext\&pid=S0100204X2009000100005\&lng= en\&nrm=iso\&tlng=pt $>$. Acesso em: 21 fev. 2014. doi: 10.1590/ S0100-204X2009000100005.

PINTHUS, M.J. Estimate of genotypic value: A proposed method. Euphytica, v.22, n.1, p.121-123, 1973. Disponível em: <http:// link.springer.com/article/10.1007/BF00021563\#page-1>. Acesso em: 21 fev. 2014. doi: 10.1007/BF00021563.

RAMALHO, M.A.P. et al. Genética quantitativa em plantas autógamas: aplicações ao melhoramento do feijoeiro. Goiânia: UFG, 1993. 271p.

SANTOS, R.C. et al. Stability and adaptability of runner peanut genoty pes based on non linear regression and AMMI analysis. Pesquisa Agropecuária Brasileira, v.47, p.1118-1124, 2012. Disponível em: <http://www.scielo.br/scielo.php?pid=S0100$204 X 2012000800012 \&$ script $=$ sci_arttext $>$. Acesso em 23 fev. 2014. doi: 10.1590/S0100-204X2012000800012.

SANTOS, R.C.et al. Recomendações técnicas para o cultivo do amendoim em pequenas propriedades agrícolas do Nordeste brasileiro. Campina Grande: Embrapa Algodão, 2006. 7p. (Boletim Técnico, 102).

SANTOS, R.C. et al. Melhoramento do amendoim. In: . $\mathbf{O}$ agronegócio do amendoim no Brasil. Campina Grande: Embrapa Algodão, 2005.p.123- 190.

SCAPIM, C.A. et al. Correlations between the stability and adaptability statistics of popcorn cultivars. Euphytica, v.174, n.2, p.209-218, 2010. Disponível em: <http://ink.springer.com/ article/10.1007\%2Fs10681-010-0118-y\#page-1>. Acesso em: 23 fev. 2014. doi: 10.1007/s10681-010-0118-y.

SILVA FILHO, J.L. et al. Comparação de métodos para avaliar a adaptabilidade e estabilidade produtiva em algodoeiro. Pesquisa Agropecuária Brasileira, v.43, n.3, p.349-355, 2008. Disponível em: $<$ http://www.scielo.br/scielo.php?script=sci_arttext\&pid=S0100$204 X 2008000300009 \& \operatorname{lng}=$ en\&nrm=iso\&tlng $=\mathrm{pt}>$. Acesso em: 23 fev. 2014. doi: 10.1590/S0100-204X2008000300009.

SILVA, W.C.J.; DUARTE, J.B. Métodos estatísticos para estudo de adaptabilidade e estabilidade fenotípica em soja. Pesquisa Agropecuária Brasileira, v.41, n.1, p.23-30, 2006. Disponível em: $<$ http://www.scielo.br/scielo.php?script=sci_arttext\&pid=S0100$204 X 2006000100004 \& \operatorname{lng}=\mathrm{en} \& n \mathrm{~nm}=\mathrm{iso} \& \mathrm{t} \operatorname{lng}=\mathrm{pt}>$. Acesso em: 23 fev. 2014. doi: 10.1590/S0100-204X2006000100004.

WRICKE, G. Zur Berechnung derÖkovalenzbei Sommerweizenund Hafer. Pflanzenzuchtungv, v.52, p.127-138. 1965. 